\title{
MEMS deformable mirrors in astronomical adaptive optics
}

\author{
T. Bifano ${ }^{1}$, S. Cornelissen ${ }^{2}$, and P. Bierden ${ }^{2}$ \\ ${ }^{1}$ Boston University Photonics Center, Boston MA 02215 USA \\ ${ }^{2}$ Boston Micromachines Corporation, Cambridge MA 02138 USA
}

\begin{abstract}
We report on the development of microelectromechanical (MEMS) deformable mirrors designed for ground and space-based astronomical instruments using adaptive optics. These light-weight, low power deformable mirrors will have an active aperture of up to $25.2 \mathrm{~mm}$ consisting of thin silicon membrane mirror supported by an array of up to 4096 electrostatic actuators exhibiting no hysteresis and sub-nanometer repeatability. The continuous membrane deformable mirrors, coated with a highly reflective metal film, are capable of up to $4 \mu \mathrm{m}$ of stroke, have a surface finish of $<10 \mathrm{~nm}$ RMS with a fill factor of $99.8 \%$. The segmented device has a range of motion of 1 um of piston and 600 arc-seconds of tip/tilt simultaneously and a surface finish of $5 \mathrm{~nm}$ RMS. Presented in this paper are device characteristics and performance results for these devices.
\end{abstract}

\section{Motivation for high-resolution wavefront correction}

Most large ground based telescopes now employ AO as an essential and enabling tool for highresolution imaging. Though recent progress in AO for current and future large telescopes has been technologically exciting, its impact on astronomical science remains modest to date. AO technology is still inadequate to compensate for the larger wavefront errors and shorter atmospheric coherence lengths associated with visible wavelength observation. Its simplest implementation allows only a narrow field of compensation, and more ambitious instrument concepts are limited by the cost, size, and complexity of AO components, especially the DM. A critical assessment of AO technology was funded by the National Science Foundation several years ago, resulting in a 2008 report entitled $\underline{A}$ Roadmap for the Development of United States Astronomical Adaptive Optics. The roadmap identifies single major goal for wavefront correction: "Development of scalable, cost-effective DM technologies" and recommends a high-priority research investment to achieve this goal: "High stroke, high actuator count [deformable] mirrors to enable correction at high spatial frequencies over narrower fields of view."

The coming generation of ELTs, will be the first to be designed with AO at the outset. Since the benefits of AO increase nonlinearly with telescope aperture, a phenomenon sometimes called $\mathrm{D}^{4}$ scaling, AO will be essential for many ELT science goals [1,2]. Promising AO instruments that will require new DMs include multi-conjugate adaptive optics (MCAO), multi-object adaptive optics (MOAO), and extreme adaptive optics (ExAO) [3]. MCAO employs two or more guide stars to enable tomographic wavefront error sensing, and then two or more deformable mirrors in series to correct those errors. The result is a wider corrected field of view [4]. This technique was recently used to produce the sharpest whole-planet (Jupiter) picture ever taken from the ground [5]. MOAO is an instrument concept that also uses multiple guide stars and multiple deformable mirrors [6]. However in MOAO, many DMs would be used in parallel to apply independent corrections for the turbulence-induced wavefront distortions in separate subregions of interest, allowing high-resolution imaging of small science objects distributed across a large field of view. ExAO refers to a family of planned ground-based instruments intended for observation of exoplanets using ultra high resolution

This is an Open Access article distributed under the terms of the Creative Commons Attribution-Noncommercial License, which permits unrestricted use, distribution, and reproduction in any noncommercial medium, provided the original work is properly cited. 
DMs having thousands or tens of thousands of actuators. In addition to exoplanet detection, an ExAO system will be capable of characterizing dust disks around stars and could allow high Strehl ratio imaging at visible wavelengths [7-9].

\section{Wavefront correction technology}

Conventional macroscale DMs, made with either piezo-bimorphs or stacked piezo-actuators are limited as high-resolution astronomical telescope wavefront correctors. Their manual assembly makes it costly and difficult to scale to devices with large actuator count, and their large mass results in complexity in integration on telescopes. Their large power requirements and bulky electronics drivers compound these limitations. Two currently active projects for high-resolution DMs will employ conventional DMs. The first, PALM 3000, is an upgrade to the Palomar AO system on the 5.1 meter Hale telescope. The high-resolution DM for that instrument will be a 3388 actuator DM manufactured by Northrop Xinetics, with usable stroke of $\sim 1.2 \mu \mathrm{m}$. Its two full height racks of drivers dissipate $4 \mathrm{~kW}$, and cable volume constraints compelled instrument designers to locate these at the Cassegrain focus, requiring liquid cooling systems [10]. The second, a CILAS DM with 3000 actuators is planned for use in the Thirty Meter Telescope. This device is a scale-up based on the Cilas SPHERE DM with 1377 actuators that delivered by CILAS to the European Southern Observatory in 2007 . It will weigh $\sim 400 \mathrm{~kg}$. Costs for such conventional DM systems are of order $\sim$ \$1500/actuator.

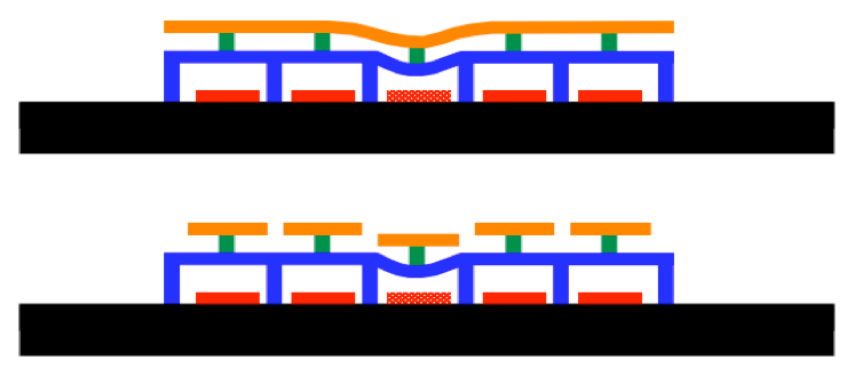

Figure 1: Schematic cross section of continuous and segmented MEMS DMs. The mirror is connected by posts to an array of electrostatic actuators. Actuator deflection is approximately proportional to the square of voltage applied to the rigid electrodes on the wafer substrate.

Over the past decade, our research group at Boston University (BU), in partnership with Boston Micromachines Corporation (BMC), have pioneered design, fabrication, and control of a promising new class of deformable mirrors (DMs) for adaptive optics (AO) based on microelectromechanical systems (MEMS) technology. The DM architecture, illustrated in Figure 1, is based on a scalable array of parallel plate electrostatic actuators, fabricated in silicon through semiconductor batch processing. Each square actuator plate is rigidly connected to the substrate along two of its edges, and is suspended above an addressable electrode. Voltage applied to that electrode imposes an electrostatic attractive force on the electrically grounded actuator plate, causing it to bend toward the substrate in proportion to the square of applied voltage. Each actuator plate has a central post connected to a continuous or segmented mirror layer. The design and manufacturing approaches developed in our MEMS DM research offer inherent advantages. In design, the actuation mechanism is repeatable to sub-nanometer precision, exhibits no hysteresis, and is unaffected by billions of cycles of operation. It is also compact. In some cases this can be a liability, since the Lagrange invariant imposes constraints on the practical demagnification achievable in a large telescope as the beam propagates from the primary to the optically conjugate DM. However, when the smaller MEMS DM can be tolerated (e.g. narrow filed MOAO and ExAO applications) it reduces system 
weight and power by more than two orders of magnitude to $\sim 10 \mathrm{~g}$ and $\sim 10 \mathrm{~mW}$ per channel, respectively. In Manufacturing, an advantage is that the devices are batch produced many wafers at a time, so that while development costs are high, commercial production and replication costs are low. The MEMS DM systems (including driver) commercialized by BU/BMC cost about $\$ 150 /$ actuator, which is one tenth that of conventional DMs. In addition, Multiple devices can be produced on each wafer, allowing broad parameter variation in a single batch production cycle. This accelerates research and prototyping. In summary, MEMS-DMs offer the rare opportunity to develop technology that will be both more economical and more capable than the state-of-the-art.

In addition to higher resolution, ELT AO instruments will challenge other DM performance limits, with demands for larger stroke, nanometer-scale open loop control repeatability, and flawless actuator arrays. MEMS DM technology is particularly compelling for the latter two. MOAO will require open loop control of the DM since corrections made by the DM are not accessible to the wavefront sensor. That makes it important that the DM can be shaped to the required precision in a single step, something that is difficult or impossible to do using conventional DMs but achievable with MEMS. Open-loop operation also enables separate correction of tip/tilt stars in a laser guide star system, which significantly improves sky coverage [9]. We recently implemented a computationally simple and inherently fast open loop control algorithm on a BU 140 actuator DM. Shapes at the limit of achievable mirror spatial frequencies were achieved with less than $15 \mathrm{~nm}$ RMS error [11]. Others have also implemented open loop AO control with BU/BMC mirrors, demonstrating comparable results $[12,13]$. That open loop control architecture and the BU/BMC DM was demonstrated in the first civilian use of MEMS-based astronomical AO in the Villages project led by Gavel in the past year [14, 15]. ExAO on ELTs will require DMs with many thousands of actuators, shape control to within a few nanometers and $100 \%$ actuator yield. MEMSDMs made using the BU heritage design have shown promise in this regard, with a generation of flawless 1024 actuator DMs now routinely available commercially, and a 4096 DM that is currently being developed by BMC for the Gemini Planet Imager (GPI).

\section{MEMS DM state-of-the-art}

Our research effort has produced segmented and continuous mirrors with 32 to 4096 actuators, mirror apertures from $1.5 \mathrm{~mm}$ to $26 \mathrm{~mm}$, and stroke from $2 \mu \mathrm{m}$ to $8 \mu \mathrm{m}$. On the basis of this research and development, BMC now produces commercial products that include the MultiDM, with 140 actuators and a USB controlled driver, and the KiloDM and KiloSLM, continuous and segmented versions of a 1024 actuator device that features $30 \mathrm{kHz}$ frame rate. We have also manufactured unique DMs for laser communication applications, space-based reconnaissance applications, and biological imaging applications.

The focus of our development has been guided by astronomical science demands for additional stroke and larger numbers of actuators. Figure 2 depicts graphically the history and future plans for BU MEMS DMs with respect to those critical parameters. It is important to note that the peak values obtained in stroke and actuator count have not been obtained simultaneously. The largest stroke DMs $(8 \mu \mathrm{m})$ were manufactured on 140 actuator devices, while the largest actuator count devices (4096) featured $3.5 \mu \mathrm{m}$ stroke.

Measurements made on 1024 actuator MEMS DMs by the UCSC-LAO demonstrated sub-nanometer repeatability, sub-nanometer stability, and sub-nanometer hysteresis over a wide range of operating conditions [16]. These characteristics, are unachievable using conventional DMs and might enable unprecedented AO instrumentation. With closed loop control, the DM has been flattened in the LAO testbed to within a $0.54 \mathrm{~nm}$ in its controllable band of spatial frequencies (and 13nm RMS overall) [17] . 


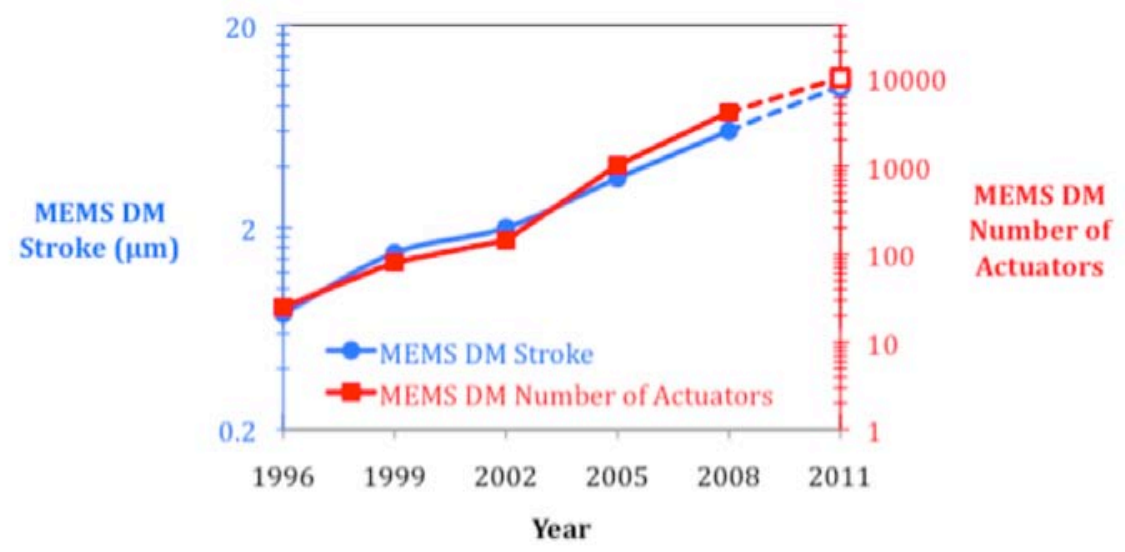

Figure 2: A history of DM peak achievable stroke and actuator count for the MEMS DMs pioneered at BU. Data correspond to fully functional deformable mirror devices with $>99 \%$ working actuators $(100 \%$ for devices with 1024 or fewer actuators) and optical surface quality. Results for 2011 are projected.

\section{Astronomical AO with MEMS DMs}

Based on heritage device designs and process science developed at BU and BMC, we recently produced a 4096 actuator 26mm aperture MEMS-DM for GPI, a new planet imaging instrument [18, 19]. An engineering grade mirror, the first of its kind, has been delivered and is being evaluated. A science grade mirror will be delivered early in 2010. Figure 3 illustrates some preliminary results from the engineering grade mirror produced for GPI. The compact ceramic package strains the boundaries achievable wire-bonding, with wire bond leads individually attached through four peripheral rows of bond pads. Aside from a relatively large (700nm RMS) spherical non-flatness on the DM due to unexpected wafer bow, the device achieved target specifications. Local surface flatness was about $4 \mathrm{~nm}$ RMS over a central $500 \mu \mathrm{m}$ span, and achievable stroke exceeded $4 \mu \mathrm{m}$. Interactuator stroke exceeded $1 \mu \mathrm{m}$. Actuator yield exceeded $99 \%$.
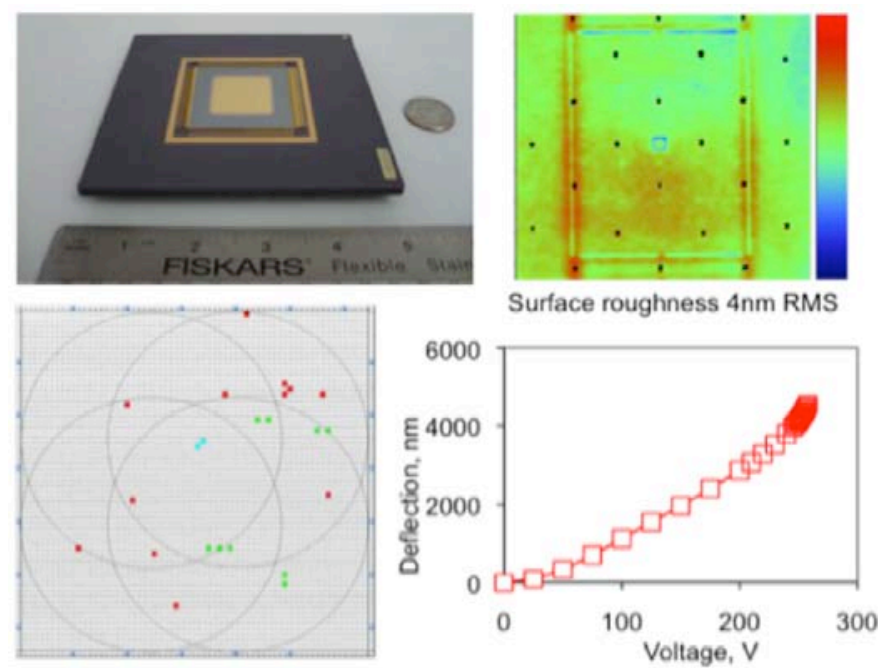

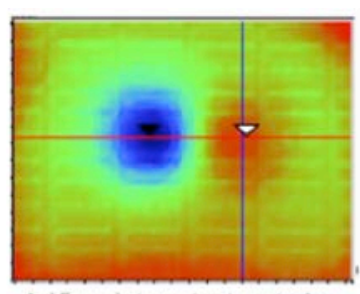

$1.15 \mu \mathrm{m}$ Interactuator stroke

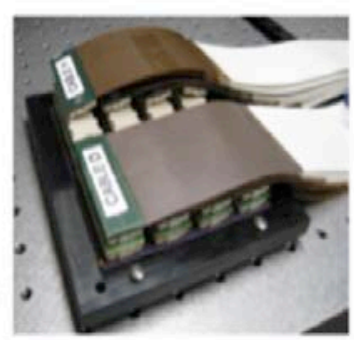

Figure 3: Engineering grade 4096 actuator MEMS DM. Clockwise from top left: Photo of the DM in its package; interferometrically measured small scale surface flatness $\sim 4 n m$ rms; interactuator stroke, 1.15 $\mu m$; Back side of package, with eight 512-wire ribbon cables; Measured voltage vs. deflection curve; Map of 20 non-yielding actuators. 

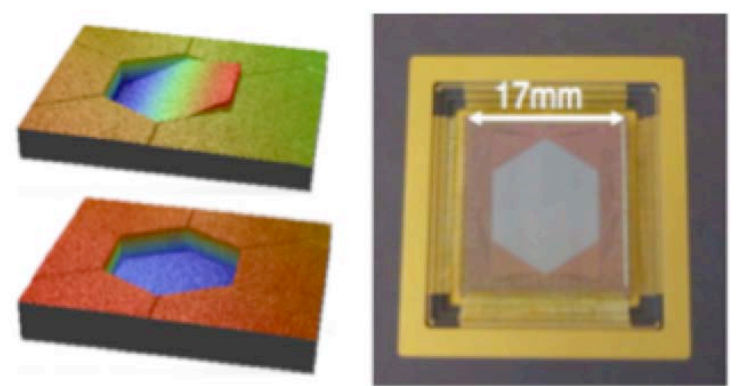

Figure 4: Ultraflat (5nm RMS) tip-tilt-piston MEMS DM with flexure relieved actuators. Left: Measured 6mrad tilt and $1 \mu m$ piston on a single segment. Right: packaged DM with 331 segments.

BMC also produced the first MEMS-DMs ever used in civilian astronomical AO, at Lick Observatory, in a project led by Don Gavel and known as the MEMS-AO/Visible Light Laser Guidestar Experiments (ViLLaGEs). The ViLLaGEs experiment represents a pioneering effort to integrate MEMS DMs an in open loop AO control system for on-sky observation [17, 20-22]. That work will be advanced by a recently awarded NSF MRI grant to Gavel, which will support integration of a 1024 actuator BMC DM on the Shane telescope AO system.

BU and BMC collaborated recently on a project to deliver advanced DMs for space-based astronomical imaging supported by NASA JPL [23]. That work involved development of a segmented tip-tilt-piston mirror for a visible nulling coronagraph envisioned as part of the Terrestrial Planet Finder (TPF) program. Specifications for the mirror required advances in both design and fabrication processes, to achieve $5 \mathrm{~nm}$ RMS flatness on the segments and to ensure less than $2 \mathrm{~nm}$ bending of the $600 \mu \mathrm{m}$ diameter segments during tilt actuation. The innovative design included a flexure at the base of each of the three attachment posts connected to each mirror segment, and a $15 \mu \mathrm{m}$ thick post-polished silicon layer for the mirror, grown in a new epitaxial deposition process that was co-developed with a MEMS foundry. The device (Figure 4) was delivered to JPL this year. It was successfully tested at JPL's High Contrast Imaging Testbed, and results led to a NASA contract to produce an upgraded DM with 1027 segments (3071 actuators) in the coming year.

\section{Acknowledgements}

Professor Bifano acknowledges a financial interest in Boston Micromachines Corporation.

\section{References}

1. Gavel D, SPIE MEMS Adaptive Optics III, San Jose, CA, USA, SPIE, [7209], 72090E-72095, 2009.

2. Ellerbroek B, Adkins S, Andersen D, Atwood J, Boyer C, Byrnes P, Conan R, Gilles L, Herriot G, Hickson P, Hileman E, Joyce D, Leckie B, Liang M, Pfrommer T, Sinquin JC, Veran JP, Wang LQ, Welle P, Adaptive Optics Systems, Pts 1-3, [7015], R150, 2008.

3. Crampton D, Simard L, Ground-based and Airborne Instrumentation for Astronomy, Orlando, FL, USA, SPIE, [6269], 62691T-62615, 2006.

4. Ragazzoni R, Marchetti E, Valente G, Nature, [403], 54-56, 2000.

5. Wong MH, Marchis F, Marchetti E, Amico P, Tordo S, Bouy H, Pater Id, 40th Annual Meeting of the Division for Planetary Sciences, Ithaca, NY, American Astronomical Society, 2008.

6. Dekany RG, Britton MC, Gavel DT, Ellerbroek BL, Herriot G, Max CE, Veran J-P, SPIE Advancements in Adaptive Optics, Glasgow, Scotland, United Kingdom, SPIE, [5490], 879-890, 2004. 
7. Evans JW, Morzinski K, Reza L, Severson S, Poyneer L, Macintosh BA, Dillon D, Sommargren G, Palmer D, Gavel D, Olivier S, Techniques and Instrumentation for Detection of Exoplanets II, San Diego, CA, USA, SPIE, [5905], 59050Y-59058, 2005.

8. Evans JW, Morzinski K, Severson S, Poyneer L, Macintosh B, Dillon D, Reza L, Gavel D, Palmer D, Olivier S, Bierden P, SPIE MEMS/MOEMS Components and Their Applications III, San Jose, CA, USA, SPIE, [6113], 61130I-61136, 2006.

9. Macintosh B, Graham J, Palmer D, Doyon R, Gavel D, Larkin J, Oppenheimer B, Saddlemyer L, Wallace JK, Bauman B, Erikson D, Poyneer L, Sivaramakrishnan A, Soummer R, Veran JP, Comptes Rendus Physique, [8], 365-373, 2007.

10. Bouchez AH, Dekany RG, Angione JR, Baranec C, Bui K, Burruss RS, Crepp JR, Croner EE, Cromer JL, Guiwits SR, Hale DDS, Henning JR, Palmer D, Roberts JE, Troy M, Truong TN, Zolkower J, Astronomical and Space Optical Systems, San Diego, CA, USA, SPIE, [7439], 74390H-74398, 2009.

11. Stewart JB, Diouf A, Zhou YP, Bifano TG, Journal of the Optical Society of America a-Optics Image Science and Vision, [24], 3827-3833, 2007.

12. Morzinski KM, Harpsoe KBW, Gavel DT, Ammons SM, SPIE MEMS Adaptive Optics, San Jose, CA, USA, SPIE, [6467], 64670G-64610, 2007.

13. Curtis RV, Glenn T, Rodolphe C, Celia B, Adaptive Optics: Methods, Analysis and Applications, Optical Society of America, AOThD4, 2009.

14. Grigsby B, Lockwood C, Baumann B, Gavel D, Johnson J, Ammons SM, Dillon D, Morzinski K, Reinig M, Palmer D, Severson S, Gates E, Advanced Optical and Mechanical Technologies in Telescopes and Instrumentation, Marseille, France, SPIE, [7018], 701841-701812, 2008.

15. Gavel D, Severson S, Bauman B, Dillon D, Reinig M, Lockwood C, Palmer D, Morzinski K, Ammons M, Gates E, Grigsby B, MEMS Adaptive Optics II, San Jose, CA, USA, SPIE, [6888], 688804-688807, 2008.

16. Morzinski KM, Gavel DT, Norton AP, Dillon DR, Reinig MR, MEMS Adaptive Optics II, San Jose, CA, USA, SPIE, [6888], 68880S-68812, 2008.

17. Evans JW, Macintosh B, Poyneer L, Morzinski K, Severson S, Dillon D, Gavel D, Reza L, Optics Express, [14], 5558-5570, 2006.

18. Macintosh B, Graham J, Palmer D, Doyon R, Gavel D, Larkin J, Oppenheimer B, Saddlemyer L, Wallace JK, Bauman B, Evans J, Erikson D, Morzinski K, Phillion D, Poyneer L, Sivaramakrishnan A, Soummer R, Thibault S, Veran J-P, Advances in Adaptive Optics II, Orlando, FL, USA, SPIE, [6272], 62720L-62712, 2006.

19. Cornelissen SA, Bierden PA, Bifano TG, Lam CV, Journal of Micro/Nanolithography, MEMS and MOEMS, [8], 031308-031308, 2009.

20. Morzinski KM, Macintosh BA, Dillon D, Gavel D, Palmer D, Norton A, Adaptive Optics Systems, Marseille, France, SPIE, [7015], 70153N-70112, 2008.

21. Poyneer LA, Bauman B, Macintosh BA, Dillon D, Severson S, Optics Letters, [31], 293-295, 2006.

22. Poyneer LA, Dillon D, Thomas S, Macintosh BA, Applied Optics, [47], 1317-1326, 2008.

23. Stewart JB, Bifano TG, Cornelissen S, Bierden P, Levine BM, Cook T, Sensors and Actuators a-Physical, [138], 230-238, 2007. 